\title{
Changes in the hormone and lipid profile of obese adolescent Saudi females with acne vulgaris
}

\author{
K.O. Abulnaja \\ Biochemistry Department, Faculty of Science, King Abdulaziz University, Jeddah, Saudi Arabia \\ Correspondence to: K.O. Abulnaja, Biochemistry Department, Faculty of Science, King Abdulaziz \\ University, P.O. Box 80203, Jeddah 21589, Saudi Arabia \\ E-mail: <kabulnaja@yahoo.com> or <kabualnaja@kau.edu.sa>
}

\begin{abstract}
Acne vulgaris is a multifactorial disease affecting a majority of the adolescent population. The objective of this study was to test for a correlation between fasting serum lipid profiles and levels of testosterone, insulin, leptin, and interleukin 1- $\beta$ (IL-1 $\beta$ ) and the incidence of severe acne vulgaris in obese adolescent females. Four groups of adolescent females were studied: obese with acne, obese without acne, non-obese with acne, and non-obese without acne. Obese females with acne, compared to obese females without acne and non-obese subjects, had significantly higher serum triglycerides, low-density lipoprotein cholesterol and apolipoprotein-B (apo-B) (mean \pm SD: $197 \pm 13.7$ vs $171 \pm 11.5,128 \pm 8.3$ vs $116 \pm 7.7,96 \pm 13.7$ vs $85 \pm 10.3 \mathrm{mg} / \mathrm{dL}$, respectively) but significantly lower high-density lipoprotein cholesterol and apo-A1 levels (40 \pm 3.3 vs $33 \pm 3.5$ and $126 \pm 12$ vs $147 \pm 13 \mathrm{mg} / \mathrm{dL}$ ). Serum testosterone, insulin and leptin levels were significantly higher in obese subjects with or without acne compared to non-obese females with or without acne ( $3 \pm 0.5$ vs $2.1 \pm 0.47,15.5 \pm 3.3$ vs $11.6 \pm 3,0.9 \pm 0.2$ vs $0.6 \pm 0.15 \mathrm{nmol} /$ $\mathrm{mL}$, respectively). Serum IL-1 $\beta$ was significantly elevated in obese and non-obese subjects with acne compared to subjects without acne; in those without acne, these levels were higher in obese than non-obese subjects $(2.4 \pm 0.2,1.4 \pm 0.1$ vs $1.8 \pm 0.12$ and $1.3 \pm 0.11 \mathrm{pg} / \mathrm{mL}$, respectively). Our results indicate that there is a relationship between obesity (BMI >27) and acne. By early recognition, the etiology and treatment protocol of acne may prevent unwanted conditions.
\end{abstract}

Key words: Acne vulgaris; Obesity; Testosterone; Adolescent females; Serum IL-1 $\beta$

Received April 27, 2008. Accepted April 8, 2009

\section{Introduction}

Adolescence is the transitional stage of physical and mental human development that occurs between childhood and adulthood. This transition involves biological (i.e., pubertal), social and psychological changes, with the biological or physiological ones being the easiest to measure objectively. Historically, puberty has been heavily associated with teenagers and the onset of adolescent development (1). However, the onset of puberty has been increasing in preadolescence or has extended beyond the teenage years, making adolescence less simple to discern (2). Adolescence essentially begins when physiologically normal puberty starts and ends when the person develops an adult identity and behavior. This period of development corresponds roughly to the period between the ages of 10 and 19 years. Certainly, some adolescents take a few years longer to develop their adult identity and behavior, sometimes into their early to mid-twenties.

The end of adolescence and the beginning of adulthood vary by country as well as by function, since even within a single country there will be different ages at which an individual is considered mature enough to be entrusted with particular tasks, such as driving a vehicle, having sexual relations, serving in the armed forces, voting, or marrying. Also, adolescence is usually accompanied by an increased independence allowed by the parents or legal guardians and less supervision, contrary to the preadolescence stage (2).

Acne vulgaris is a common disease that affects the majority of the adolescent population and a large number of young adults (3). Facial acne is the cause for many visits 
to primary care physicians and dermatologists, which can involve enormous costs (4). Little is known about the natural history of acne, despite its prevalence and its toll in scarring and psychological damage (5). Acne appears very early in puberty during the preteen years, often before menarche in girls (6). Because many adolescents have acne, it is difficult to predict which individuals are prone to severe cases. The four primary factors determining its development are abnormal follicular epithelial desquamation, hyperactivity of the sebaceous glands, proliferation of Propionibacterium acnes, and follicular inflammation (7).

Both clinical observations and experimental evidence confirm the importance of androgens in the pathophysiology of acne. The major androgens that interact with the androgen receptor are testosterone and dihydrotestosterone (8). An association between diet and acne has long been postulated, but studies that have examined the impact of diet on acne have produced controversial results. Some have indicated that diets high in carbohydrates and fat worsen acne, while others did not detect this relationship (9). Hyperinsulinemic diets were also thought to be an environmental factor in the development of acne since they influence follicular epithelial growth, keratinization and androgen-mediated sebum secretion (10).

Some experiments have demonstrated that leptin directly controls the secretory activity of human ovarian cells, with an interrelationship between leptin and insulin growth factor (11). In light of the variable stress factors affecting the neuroendocrine axis during adolescence that occur in tandem with the modern lifestyle, a specific imbalance of neurotransmitters and hormones develops that could influence a complex sequence of behavioral changes (12).

Emotions, on the other hand, may combine the psychological and physiological features observed during external environmental challenges and could be co-linked with subcellular elements that are geared toward maintaining a constant internal environment. Thus, the presence of excess adipose tissue, which is maladaptive, could induce lipid peroxidation, which affects the inflammatory processes of innate immunity that are part of the response to acne vulgaris pathogenesis (13).

The present study was designed to evaluate the relationship of the lipid profile, endocrine levels (testosterone, leptin and insulin) and the immunoinflammatory response (interleukin 1- $\beta, \mathrm{IL}-1 \beta$ ) to the incidence of acne vulgaris in obese and non-obese Saudi adolescent females.

\section{Material and Methods}

The medical records of King Abdul Aziz University Hospital Medical Center were reviewed to identify all fe- male patients who had been submitted to an initial "hirsutism panel". This panel included tests ordered for female adolescents with a clinical suspicion of hyperandrogenism based on the presence of one or more of the following symptoms: irregular periods, hirsutism, acne, and obesity. Subjects were included in the study if they attended at least one visit and had at least one abnormal parameter in the hirsutism panel tests. Gynecologic services were provided by a faculty obstetrician-gynecologist and by three pediatric and adolescent gynecology fellows. Primary clinicians of the Teen Health Center (adolescent medicine specialists and nurse practitioners) also provided gynecologic services. All subjects were outpatients of the Dermatology Unit of the University Hospital. The study protocol was approved by the local Ethics Committee and a written informed consent was obtained from each subject.

The study was conducted on a total of 60 adolescent females aged 16 to 22 years divided into four groups of 15 subjects each: obese with severe acne, obese without acne, non-obese with acne, and non-obese without acne. The period of study was from February to May 2007. They attended the dermatology units for skin and face care and hair removal techniques, including plucking, use of depilatories, shaving, bleaching, laser hair removal, and electrolysis.

Subjects with up to 10 lesions were defined as having mild acne, cases with 11 to 25 lesions were defined as having moderate acne, and subjects with more than 25 lesions were defined as having severe acne. This classification was in accordance with the International Consensus Conference on Acne Classification System. Obesity was defined as a body mass index $(\mathrm{BMI})>27 \mathrm{~kg} / \mathrm{m}^{2}$ (12) and waist-to-hip ratio $>0.8(14,15)$.

Clinical examination showed that they were non-diabetic and free from any neuroendocrine disorders, with no history of hypertension, liver, kidney or heart disease, and that they were not under any treatment for acne to avoid the effect of other endocrine disturbance.

Expert opinions vary as to the necessary criteria for the diagnosis of polycystic ovary syndrome (PCOS). Hyperandrogenism should be considered in female adolescents if irregular bleeding is accompanied by findings on physical examination such as hirsutism, moderate-severe acne, obesity, or acanthosis nigricans. Early identification and treatment can potentially alter a young woman's future health risks.

Fasting blood samples $(10 \mathrm{~mL})$ were taken from all subjects for serum separation and for the following analyses: lipoprotein profile involving total cholesterol, low-density lipoprotein cholesterol (LDL-C) and high-density lipoprotein cholesterol (HDL-C) assessed by the method of Wilson and Spiger (16); triglycerides, according to Fossati 
and Prencipe (17); apolipoprotein-A1 (apo-A1) and apolipoprotein-B (apo-B) by the immunoturbidimetric method of Reipponen et al. (18).

Hormonal milieu, including insulin (19), testosterone $(20,21)$ and leptin $(22)$, was assessed by radioimmunoassay using a commercial kit from Diagnostic Products Corporation, USA. IL-1 $\beta$ was determined by ELISA (23).

\section{Statistical analysis}

Data are reported as means \pm SD. Statistical comparisons were made using the Student $t$-test and the nonparametric chi-square and Kruskal-Wallis tests. A P value of less than 0.05 was accepted as significant. The Pearson product-moment correlation coefficient was also used for analysis (SPSS version 7).

\section{Results}

The sociodemographic data of the subjects, presented in Table 1, show that the ages of the adolescent females selected for this study were not different among groups. $\mathrm{BMI}$ of the obese females with or without acne was significantly higher than non-obese patients with or without acne $(P<0.05)$, as expected.

Table 1 also shows that obese females with acne had significantly higher serum levels of triglycerides, LDL-C and apo-B ( $P<0.05$ for each) and significantly lower $(\mathrm{P}<$ 0.05) HDL-C and apo-A1 levels compared to obese females without acne. Obese females with acne also had significantly higher levels of triglycerides and LDL-C and significantly lower apo-A1 levels $(P<0.01)$ than nonobese females with acne. Apo-B levels did not differ signifi- cantly between obese and non-obese patients with acne.

Hormonal differences are also presented in Table 1. The levels of serum testosterone, insulin, leptin, and IL-1 $\beta$ $(\mathrm{P}<0.05, \mathrm{P}<0.01, \mathrm{P}<0.05$, and $\mathrm{P}<0.05)$ in obese subjects with acne were significantly higher than obese subjects without acne. The levels of testosterone, insulin and leptin in the obese subjects with or without acne was significantly higher than the non-obese subjects with or without acne $(P<0.05, P<0.05$, and $P<0.001)$.

There was a positive correlation between testosterone and leptin $(r=0.61)$ and between testosterone and triglycerides $(r=0.59)$ in obese females with acne.

\section{Discussion}

Acne is a multifactorial disease. The four primary factors determining its development are abnormal follicular epithelial desquamation, hyperactivity of the sebaceous glands, proliferation of Propionibacterium acne, and perifollicular inflammation. Clinically, acne is also a pleomorphic disease, characterized by a mixture of inflammatory and noninflammatory lesions (5). In the present study, we examined the lipid profiles and the levels of apoproteins, hormones and inflammatory cytokines in relation to obesity in adolescent females with acne.

Adolescents with very irregular cycles, particularly with signs of excess androgen, should be evaluated further with laboratory measurement of androgens. A menstrual calendar is of great value since it may be difficult to obtain a reliable menstrual history from many teens. Of importance is a careful and complete physical examination of these young women (4).

Table 1. Characteristics of obese and non-obese adolescent females with or without acne.

\begin{tabular}{lccccc}
\hline \multirow{2}{*}{ Parameters } & \multicolumn{2}{c}{ Obese } & & \multicolumn{2}{c}{ Non-obese } \\
\cline { 2 - 3 } \cline { 5 - 6 } & With acne & Without acne & & With acne & Without acne \\
\hline Age & $18 \pm 1.3$ & $20 \pm 1.9$ & & $17 \pm 1.4$ & $18 \pm 1.6$ \\
Body mass index & $30 \pm 2.1^{+}$ & $29 \pm 1.7^{+}$ & & $26 \pm 2.1$ & $25 \pm 1.9$ \\
Triglycerides $(\mathrm{mg} / \mathrm{dL})$ & $197 \pm 13.7^{*+}$ & $171 \pm 11.5^{+}$ & & $180 \pm 10.4^{*}$ & $163 \pm 8.9$ \\
HDL-C $(\mathrm{mg} / \mathrm{dL})$ & $40 \pm 3.3^{*}$ & $33 \pm 3.5^{+}$ & & $42 \pm 3.0^{*}$ & $51 \pm 4.0$ \\
LDL-C $(\mathrm{mg} / \mathrm{dL})$ & $128 \pm 8.3^{*+}$ & $116 \pm 7.7^{+}$ & & $117 \pm 8.1^{*}$ & $102 \pm 6$ \\
apo-A1 $(\mathrm{mg} / \mathrm{dL})$ & $126 \pm 12^{*+}$ & $147 \pm 13^{+}$ & & $170 \pm 14$ & $163 \pm 11$ \\
ApoB $(\mathrm{mg} / \mathrm{dL})$ & $96 \pm 13.7^{*}$ & $85 \pm 10.3^{+}$ & & $91 \pm 13.1^{*}$ & $63.1 \pm 12.1$ \\
Testosterone $(\mathrm{nmol} / \mathrm{mL})$ & $3 \pm 0.5^{*+}$ & $2.1 \pm 0.47^{+}$ & & $2.1 \pm 0.3^{*}$ & $1.5 \pm 0.4$ \\
Insulin $(\mathrm{nmol} / \mathrm{mL})$ & $15.5 \pm 3.3^{*+}$ & $11.6 \pm 3^{+}$ & & $7.2 \pm 2.5$ & $7.8 \pm 2$ \\
Leptin $(\mathrm{nmol} / \mathrm{mL})$ & $0.9 \pm 0.2^{*+}$ & $0.6 \pm 0.15^{+}$ & & $0.4 \pm 0.12$ & $0.29 \pm 0.1$ \\
Interleukin 1- $\beta(\mathrm{pg} / \mathrm{mL})$ & $2.4 \pm 0.2^{*}$ & $1.4 \pm 0.1^{+}$ & & $1.8 \pm 0.12^{*}$ & $1.3 \pm 0.11$ \\
\hline
\end{tabular}

Data are reported as means $\pm S D$ for $N=15$ females in each group. ${ }^{*} P<0.05$, obese females with acne $v s$ without acne and non-obese females with acne $v s$ without acne. ${ }^{+} P<0.05$, obese females with or without acne $v s$ nonobese females with or without acne. The $t$-test was used for statistical analysis. 
It is known that bacterial hydrolases convert some of the triglycerides into free fatty acids on the skin surface (24); however, there is also evidence indicating that sebaceous glands can also synthesize considerable amounts of free fatty acids. A number of studies have confirmed changes in the lipid composition of sebum that are associated with age or with sebaceous gland activity. In addition, the effect of androgens on sebaceous cell proliferation and differentiation is dependent on the origin of the sebaceous glands; for example, facial sebaceous glands are more sensitive to androgens (24).

Adolescent females with any degree of hyperandrogenism are at risk for other metabolic derangements. In the present study, a significant elevation in the levels of serum TG, LDL-C and HDL-C was observed in obese adolescents with acne compared to without acne.

Based on the present findings in obese acne cases, there appears to be a negative correlation between the assessed alterations in lipid parameters, including increased triglyceride, LDL-C and apo-B and decreased HDL-C and apo-A1 (Table 1). This suggests that increased androgen levels lead to stimulation of the production of sebum, and the consequent proliferation of Propionibacterium generates inflammation, as was observed here in obese subjects with acne (25).

Nagy et al. (26) studied hyperandrogenic women with severe acne to determine the effect of ketoconazole (KTZ), an oral synthetic antifungal imidazole derivative that inhibits gonadal and adrenal steroidogenesis, on lipids, lipoprotein cholesterols, apolipoproteins, endogenous sex steroid hormones, and their interactions. KTZ therapy reduced cholesterol and LDL-C, increased apo-A, decreased apo-B and reduced the ratio of LDL-C to HDL-C compared to the untreated control. The reduction in total cholesterol and LDL-C appears to occur as a result of the increase in estradiol and possibly the decrease in free testosterone.

Hyperandrogenism is a common cause of irregular menses in adolescent females, and affects $5-7 \%$ of adult women (26). Girls and women with PCOS or hyperandrogenism may present a variety of concerns and symptoms, including irregular periods, excess hair growth, acne, and obesity (27). Hyperandrogenism should be considered in adolescents if irregular bleeding is accompanied by physical examination findings such as hirsutism, moderatesevere acne, obesity, or acanthosis nigricans.

In the present study, the levels of serum testosterone, insulin and leptin levels were significantly higher in obese females with acne compared to non-obese females with acne. Therefore, we suggest that testosterone may play a major role in the pathogenesis of acne. There were demographic differences between the populations of obese and non-obese females with acne. Our results indicated that there is a significant positive correlation between BMI and acne $(r=0.67)$. This conclusion needs to be verified by additional studies, especially involving obese females with acne (28).

Androgen-dependent acne in females may result from elevated serum androgen, increased cutaneous utilization of androgen or both. The ability of the pilosebaceous unit to produce sebum in response to androgen appears to be the most common mechanism leading to acne. Our data show that the androgen level in females can be used as a marker for acne. It has already been shown that sebum production is stimulated by androgen and inhibited by estrogen (29).

The results obtained revealed that obese females with acne showed higher levels of IL-1 $\beta$ compared with obese females without acne. This is in agreement with the known effects of leptin and insulin resistance on the immunologic response to inflammatory mechanisms. The production of IL-1 $\beta$, which is associated with obesity and acne, is the main function of the sebaceous glands, and the sebocytes within produce and excrete sebum. Increased sebum secretion is associated with the development of acne lesions, since sebum serves as a nutrient source (24). Other sebaceous gland functions, such as the production of proinflammatory lipids, cytokines, periglandular peptides, and neuropeptides, are also associated with the pathophysiology of acne (30). Proinflammatory cytokines/chemokines secreted by various cell types play a fundamental role in attracting neutrophils and leukocytes to the place of skin infection. Acne is able to induce the secretion of chemokines in monocytes and keratinocytes (23).

Our results are consistent with the data of Zouboulis (31), who provided evidence for the involvement of endogenous inflammatory processes in the initiation of acne. In addition, they offered logical support to the current concept of an anti-inflammatory treatment of acne. Therefore, acne vulgaris is a genuine inflammatory disease, and evidence exists to indicate that appropriate anti-inflammatory therapy has the potential to effectively treat this condition. Future compounds targeting acne vulgaris should be able to reduce proinflammatory lipids in sebum, down-regulate proinflammatory signals in the pilosebaceous unit and inhibit leukotrans-induced accumulation of inflammatory cells, as well as significantly reduce sebum production (22).

The mosaic of events of acne vulgaris appears to be affected by obesity, hormonal and immune-mediated mechanisms that potentiate the inflammatory response. We propose that reducing body weight decreases the facial lipolysis caused by bacteria and potentiates the treatment protocol. 


\section{Acknowledgments}

The author would like to thank Dr. Ahmad O. Nemah, Medical Center of King Abdul Aziz University, Jeddah,
Saudi Arabia, for providing the cases studied and the clinical investigation data.

\section{References}

1. Christie D. Clinical review: $A B C$ of adolescence. Adolescent development. www.bmj.com. Accessed March 9, 2008.

2. Hill M. UNSW embryology normal development - puberty. embryology.med.unsw.edu.au. Accessed March 9, 2008.

3. Rothman KF, Lucky AW. Acne vulgaris. In: Callen JP (Editor), Advances in dermatology. Vol. 8. St. Louis: Mosby Book; 1993. p 347-374.

4. Cypress BK. Health care of adolescents by office based physicians. Washington: US Government Printing Office. NIH publication No. 99; 1984.

5. Lucky AW, Biro FM, Huster GA, Leach AD, Morrison JA, Ratterman J. Acne vulgaris in premenarchal girls. An early sign of puberty associated with rising levels of dehydroepiandrosterone. Arch Dermatol 1994; 130: 308-314.

6. Lucky AW, Biro FM, Huster GA, Morrison JA, Elder N. Acne vulgaris in early adolescent boys. Correlations with pubertal maturation and age. Arch Dermatol 1991; 127: 210-216.

7. Leyden JJ. Therapy for acne vulgaris. N Engl J Med 1997; 336: 1156-1162.

8. Liang T, Hoyer S, Yu R, Soltani K, Lorincz AL, Hiipakka RA, et al. Immunocytochemical localization of androgen receptors in human skin using monoclonal antibodies against the androgen receptor. J Invest Dermatol 1993; 100: 663-666.

9. Thiboutot DM, Strauss JS. Diet and acne revisited. Arch Dermatol 2002; 138: 1591-1592.

10. Cordain L, Lindeberg S, Hurtado M, Hill K, Eaton SB, BrandMiller J. Acne vulgaris: a disease of Western civilization. Arch Dermatol 2002; 138: 1584-1590.

11. Stanczyk FZ. Diagnosis of hyperandrogenism: biochemical criteria. Best Pract Res Clin Endocrinol Metab 2006; 20: 177-191.

12. Saygili F, Oge A, Yilmaz C. Hyperinsulinemia and insulin insensitivity in women with nonclassical congenital adrenal hyperplasia due to 21-hydroxylase deficiency: the relationship between serum leptin levels and chronic hyperinsulinemia. Horm Res 2005; 63: 270-274.

13. Rohr UD. The impact of testosterone imbalance on depression and women's health. Maturitas 2002; 41 (Suppl 1): S25-S46.

14. Keys A, Fidanza F, Karvonen MJ, Kimura N, Taylor HL. Indices of relative weight and obesity. J Chronic Dis 1972; 25: 329-343.

15. Garrow JS, Webster J. Quetelet's index (W/H2) as a measure of fatness. Int J Obes 1985; 9: 147-153.

16. Wilson DE, Spiger MJ. A dual precipitation method for quantitative plasma lipoprotein measurement without ultracentrifugation. J Lab Clin Med 1973; 82: 473-482.

17. Fossati $P$, Prencipe L. Serum triglycerides determined colorimetrically with an enzyme that produces hydrogen peroxide. Clin Chem 1982; 28: 2077-2080.

18. Riepponen P, Marniemi J, Rautaoja T. Immunoturbidimetric determination of apolipoproteins A-1 and B in serum. Scand
J Clin Lab Invest 1987; 47: 739-744.

19. Diezfalusy E. Steroid assay by protein binding. In: Diczfalusy E (Editor), Karolinska Symposia on Research Methods in Reproductive Endocrinology. 17th edn. Stockholm: 1979. p 820-880.

20. Haffner SM, Stern MP, Hazuda HP, Pugh JA, Patterson JK. Hyperinsulinemia in a population at high risk for non-insulindependent diabetes mellitus. N Engl J Med 1986; 315: 220224.

21. Ledur A, Fitting C, David B, Hamberger C, Cavaillon JM. Variable estimates of cytokine levels produced by commercial ELISA kits: results using international cytokine standards. J Immunol Methods 1995; 186: 171-179.

22. Ma Z, Gingerich RL, Santiago JV, Klein S, Smith CH, Landt M. Radioimmunoassay of leptin in human plasma. Clin Chem 1996; 42: 942-946.

23. Tsukada M, Schroder M, Roos TC, Chandraratna RA, Reichert U, Merk HF, et al. 13-Cis retinoic acid exerts its specific activity on human sebocytes through selective intracellular isomerization to all-trans retinoic acid and binding to retinoid acid receptors. J Invest Dermatol 2000; 115: 321327.

24. Zouboulis CC. Sebaceous gland in human skin - the fantastic future of a skin appendage. J Invest Dermatol 2003; 120: xiv-xv.

25. Cedeno J, Mendoza SG, Velazquez E, Nucete H, Speirs J, Glueck CJ. Effect of ketoconazole on plasma sex hormones, lipids, lipoproteins, and apolipoproteins in hyperandrogenic women. Metabolism 1990; 39: 511-517.

26. Nagy I, Pivarcsi A, Kis K, Koreck A, Bodai L, McDowell A, et al. Propionibacterium acnes and lipopolysaccharide induce the expression of antimicrobial peptides and proinflammatory cytokines/chemokines in human sebocytes. Microbes Infect 2006; 8: 2195-2205.

27. Pivarcsi A, Nagy I, Koreck A, Kis K, Kenderessy-Szabo A, Szell M, et al. Microbial compounds induce the expression of pro-inflammatory cytokines, chemokines and human beta-defensin-2 in vaginal epithelial cells. Microbes Infect 2005; 7: 1117-1127.

28. Kahsar-Miller MD, Nixon C, Boots LR, Go RC, Azziz R. Prevalence of polycystic ovary syndrome (PCOS) in firstdegree relatives of patients with PCOS. Fertil Steril 2001; 75: 53-58.

29. Kitabchi AE, Imseis RE, Bush AJ, Williams-Cleaves B, Pourmotabbed G. Racial differences in the correlation between gonadal androgens and serum insulin levels. Diabetes Care 1999; 22: 1524-1529.

30. Zouboulis CC, Piquero-Martin J. Update and future of systemic acne treatment. Dermatology 2003; 206: 37-53.

31. Zouboulis CC. Acne and sebaceous gland function. Clin Dermatol 2004; 22: 360-366. 